\title{
Green Protocol for the Synthesis of Catalyst Free Biginelli Products
}

\author{
Abdul Ghafoor ${ }^{1, *}$, Naved Sajid ${ }^{2, \text { * }}$, Muhammad Adnan ${ }^{2}$, Muhammad Naeem Khan ${ }^{1}$, Amin Abid ${ }^{1}$, \\ Ansa Madeeha Zafar ${ }^{3}$, Muhammad Ahmad ${ }^{1}$, Noreen Aslam ${ }^{3}$, Mussarat Jabeen ${ }^{3}$, Amjad Ali ${ }^{4}$, \\ Misbahul Ain Khan ${ }^{2}$ \\ ${ }^{1}$ Department of Chemistry, University of Sahiwal, Sahiwal, Pakistan \\ ${ }^{2}$ Department of Chemistry, The Islamia University of Bahawalpur, Bahawalpur, Pakistan \\ ${ }^{3}$ Department of Chemistry, The Women University, Bahawalpur, Pakistan \\ ${ }^{4}$ Department of Anatomy, CMH Institute of Medical Sciences, Multan, Pakistan
}

Email address:

agorg_ch@yahoo.com (A. Ghafoor)

${ }^{*}$ Corresponding author

\section{To cite this article:}

Abdul Ghafoor, Naved Sajid, Muhammad Adnan, Muhammad Naeem Khan, Ansa Madeeha Zafar, Muhammad Ahmad, Noreen Aslam, Mussarat Jabeen, Amjad Ali, Misbahul Ain Khan. Green Protocol for the Synthesis of Catalyst Free Biginelli Products. American Journal of Heterocyclic Chemistry. Vol. 6, No. 1, 2020, pp. 1-5. doi: 10.11648/j.ajhc.20200601.11

Received: September 5, 2019; Accepted: April 26, 2020; Published: May 28, 2020

\begin{abstract}
Applications of multicomponent reactions (MCRs) in the preparation of diverse types of heterocyclic compounds carries a unique place in chemical synthesis. Biginelli reaction, among the loop of multicomponent reactions, provide an efficient way to access of highly functionalized dihydropyrimidones (-thiones) (DHPMs). In the current study, we employed multicomponent Biginelli reaction to synthesis range of different dihydropyrimidones (-thiones) derivatives in variable yields. A solvent-free eco-friendly green protocol without any expensive catalyst has been optimized and expand to synthesize series of DHMPS in 34 to $72 \%$ yield. The optimized method proved to be successful with various types of different aromatic aldehydes, 1, 3-dicarbonyl compounds and urea (thiourea) under solvent and catalyst free condition to access dihydropyrimidones (-thiones). Different spectroscopic techniques include NMR, FTIR etc. have used to confirm the structures of compounds. Further exploration of Biginelli reaction to prepare DHPMs and related compounds is under way in the lab and will be published in due course.
\end{abstract}

Keywords: Green Chemistry, 3, 4-Dihydropyrimidones, 3, 4-Dihydropyrimidothiones, Multi Component, Synthetic Methods, One pot, Catalyst Free Reactions

\section{Introduction}

The one-pot cyclo condensation reaction of aldehydes, $\beta$ ketoesters, and urea to give 3, 4-dihydropyrimidine-2-ones was first reported in 1893 by Biginelli under strongly acidic conditions. The dihydropyrimidinone derivatives are significant due to their pharmacological properties and have emerged as vital backbones of several calcium channel blockers, antihypertensive agents, and $\alpha$-la-antagonists [1-3], Several marine alkaloids containing the dihydropyrimidine5-carboxylate core unit have been isolated [4], the most important of which are the batzelladine alkaloids which were found to be potent HIV gp-120-CD4 inhibitors [5-7].
Because of the importance of dihydropyrimidinones, the Biginelli reaction has been well studied and several variations and improvements have been realized through the discovery of milder and more competent procedures using a variety of catalysts [8-12].

Our continuous interest in Biginelli reaction has led us to explore diverse catalysts and reaction conditions which include: metal acetate [13], alcohols [14], amino acids [15], carboxylic acids [16], and aromatic and heteroaromatic acids [17]. While our current work was in progress, a short report has appeared where this reaction is claimed to be conducted on heating but details of the detailed experimental is reclusive [18]. In view of this, we would like to communicate 
our success with this reaction under solvent and catalyst free conditions.

\section{Experimental}

\subsection{Materials and Reagents}

All the chemicals and reagents used in the present study were commercially available. These were purified by usual methods of distillation (for liquids) and crystallization from appropriate solvents (for solids). Melting points were determined on a Gallenkamp melting point apparatus and are uncorrected. IR spectra were recorded on Perkin Elmer spectrum BX 1 and NMR on a Brucker $400 \mathrm{MHz}$ spectrometer using tetramethylsilane as an internal reference.

\subsection{General Procedure for the Preparation of 3, 4-Dihdropyrimidin-2 (1H)-Ones or Thiones}

Equimolar quantities of aryl aldehyde (0.02 moles), an active methylene compound ( 0.02 moles) and urea/thiourea $\left(0.02\right.$ moles) were heated in an oil bath at $120-130^{\circ} \mathrm{C}$ for 8 hours and progress of the reaction was monitored by TLC using ethyl acetate and n-hexane (1: 2) as eluent. After completion of the period the reaction mixture was cooled, precipitates formed were filtered, washed, dried and crystallized from ethanol.

According to this general procedure, the following compounds were prepared from various aldehydes. All the products of these reactions were compared with the authentic samples prepared by the literature methods and were found to be identical in all respects [M. P. (Table 1), mixed M. P, FTIR or other spectra]:

\subsubsection{Ethyl 6-methyl-2-oxo-4-phenyl-1, 2, 3, 4-tetrahydropyrimidine -5-carboxylate}

Yield: $3.75 \mathrm{~g}(72 \%)$. m. p: $200-202^{\circ} \mathrm{C}$ (Lit. m. p: 202$\left.204^{\circ} \mathrm{C}\right) .{ }^{1} \mathrm{H}$ NMR (DMSO- $\left.d_{6}\right): \delta 9.17(\mathrm{~s}, 1 \mathrm{H}, \mathrm{NH}), 7.72(\mathrm{~s}$, $1 \mathrm{H}, \mathrm{NH}), 7.21-7.32(\mathrm{~m}, 5 \mathrm{H}, \mathrm{Ar}-\mathrm{H}), 5.5(\mathrm{~s}, 1 \mathrm{H}, \mathrm{Ar}-\mathrm{CH}), 3.98$ (q, $\left.2 \mathrm{H}, \mathrm{OCH}_{2} \mathrm{CH}_{3}, J=7.2 \mathrm{~Hz}\right), 2.24\left(\mathrm{~s}, 3 \mathrm{H}, \mathrm{CH}_{3}\right), 1.08(\mathrm{t}, 3 \mathrm{H}$, $\mathrm{OCH}_{2} \mathrm{CH}_{3}, J=7.2 \mathrm{~Hz}$ ). ${ }^{13} \mathrm{C}$ NMR (DMSO- $d_{6}$ ): $\delta 165.4,152.2$, 148.4, 144.9, 128.4, 127.9, 127.8, 127.3, 126.3, 99.3, 59.2, 54.0, 17.8, 14.1. FTIR (KBr): $3414(\mathrm{NH}), 3230,3109,2936$, $1735(\mathrm{C}=\mathrm{O}$, ester $), 1680(\mathrm{C}=\mathrm{O}$, Pyrimidine $), 1620$ and 1490 (Aromatic) $\mathrm{cm}^{-1}$.

\subsubsection{Ethyl 4- (2'-methoxyphenyl)-6-methyl-2-oxo-1, 2, 3, 4-tetrahydropyrimidine-5-carboxylate}

Yield: $0.49 \mathrm{~g}(67 \%)$. m. p: $257-259^{\circ} \mathrm{C}$ (Lit. m. p: 259$\left.260^{\circ} \mathrm{C}\right) .{ }^{1} \mathrm{H}$ NMR (DMSO- $\left.d_{6}\right): \delta 9.10$ (br, s, $\left.1 \mathrm{H}, \mathrm{NH}\right), 7.14$ 7.21 (m, 2H, Ar-H), 6.82-7.09 (m, 2H, Ar-H), 7.10 (br, s, $1 \mathrm{H}, \mathrm{NH}), 5.50$ (s, $1 \mathrm{H}, \mathrm{Ar}-\mathrm{CH}), 3.93\left(\mathrm{q}, 2 \mathrm{H}, \mathrm{OCH}_{2} \mathrm{CH}_{3}, J=7.5\right.$ $\mathrm{Hz}), 3.80\left(\mathrm{~s}, 3 \mathrm{H}, \mathrm{OCH}_{3}\right), 2.30\left(\mathrm{~s}, 3 \mathrm{H}, \mathrm{CH}_{3}\right), 1.08(\mathrm{t}, 3 \mathrm{H}$, $\mathrm{OCH}_{2} \mathrm{CH}_{3}, J=7.5 \mathrm{~Hz}$ ). ${ }^{13} \mathrm{C}$ NMR (DMSO- $d_{6}$ ): $\delta 165.6,156.6$, $153.1,150.4,130.8,128.7,126.9,120.1,111.1,101.1,61.2$, 55.4, 47.9, 15.9. FTIR (KBr): $3250(\mathrm{NH}), 3100,1740(\mathrm{C}=\mathrm{O}$, ester), 1698 ( $\mathrm{C}=\mathrm{O}$, Pyrimidine), 1645 and 1510 (Aromatic) $\mathrm{cm}^{-1}$.

\subsubsection{Ethyl 4- (2', 4'-dichlorophenyl)-6-methyl-2-oxo-1, 2,} 3, 4-tetrahydropyrimidine-5-carboxylate

Yield: $0.49 \mathrm{~g}(59 \%)$. m. p: $249-251^{\circ} \mathrm{C}$ (Lit. m. p: 249 $\left.250^{\circ} \mathrm{C}\right) .{ }^{1} \mathrm{H}$ NMR (DMSO- $\left.d_{6}\right): \delta 9.33(\mathrm{~s}, 1 \mathrm{H}, \mathrm{NH}), 7.77(\mathrm{~s}, 1$ $\mathrm{H}, \mathrm{NH}), 7.31-7.57$ (m, $3 \mathrm{H}, \mathrm{Ar}-\mathrm{H}), 5.59$ (s, 1H, Ar-CH), 3.90 (q, $\left.2 \mathrm{H}, \mathrm{OCH}_{2} \mathrm{CH}_{3}, J=7.2 \mathrm{~Hz}\right), 2.29\left(\mathrm{~s}, 3 \mathrm{H}, \mathrm{CH}_{3}\right), 1.00(\mathrm{t}, 3 \mathrm{H}$, $\mathrm{OCH}_{2} \mathrm{CH}_{3}, J=7.2 \mathrm{~Hz}$ ). ${ }^{13} \mathrm{C}$ NMR (DMSO- $d_{6}$ ): $\delta 164.7,151.0$, $149.5,140.9,132.6,132.5,130.2,128.6,127.9,97.4,59.0$, 51.1, 17.6, 13.8. FTIR (KBr): $3415(\mathrm{NH}), 3219,3104,2969$, $1737(\mathrm{C}=\mathrm{O}$, ester $), 1678(\mathrm{C}=\mathrm{O}$, Pyrimidine $), 1614$ and 1425 (Aromatic) $\mathrm{cm}^{-1}$.

\subsubsection{Ethyl 6-methyl-2-oxo-4- (pyridine-3'-yl)-1, 2, 3, 4-tetrahydropyrimidine-5-carboxylate}

Yield: $0.43 \mathrm{~g}(66 \%)$. m. p: $215-217^{\circ} \mathrm{C}$ (Lit. m. p: $217-$ $218^{\circ} \mathrm{C}$ ). ${ }^{1} \mathrm{H}$ NMR (DMSO- $\left.d_{6}\right):=\delta 9.29(\mathrm{~s}, 1 \mathrm{H}, \mathrm{NH}), 7.35-$ 8.52 (m, 4H, Pyrimidine H), 7.28 (s, 1H, NH), 5.19 (s, 1H, Ar-CH), 3.98 (q, 2H, $\left.\mathrm{OCH}_{2} \mathrm{CH}_{3}, J=5.49 \mathrm{~Hz}\right), 2.26(\mathrm{~s}, 3 \mathrm{H}$, $\left.\mathrm{CH}_{3}\right), 1.06\left(\mathrm{t}, 3 \mathrm{H}, \mathrm{OCH}_{2} \mathrm{CH}_{3}, J=7.08 \mathrm{~Hz}\right) .{ }^{13} \mathrm{C}$ NMR (DMSO$\left.d_{6}\right):=\delta 164.9,151.7,148.9,148.4,147.7,139.6,133.7,123.6$, 98.1, 59.1, 52.0, 17.6, 13.8. FTIR (KBr): $3340(\mathrm{NH}), 3220$, $1735(\mathrm{C}=\mathrm{O}$, ester $), 1680(\mathrm{C}=\mathrm{O}$, Pyrimidine $), 1632$ and 1490 (Aromatic) $\mathrm{cm}^{-1}$.

\subsubsection{Ethyl 4- (3'-hydroxyphenyl)-6-methyl-2-oxo-1, 2, 3, 4-tetrahydropyrimidine-5-carboxylate}

Yield: $0.40 \mathrm{~g}(57 \%)$. m. p: $162-164^{\circ} \mathrm{C}$ (Lit. m. p: $164-$ $\left.166^{\circ} \mathrm{C}\right) .{ }^{1} \mathrm{H}$ NMR (DMSO-d $): \delta 9.12(\mathrm{~s}, 1 \mathrm{H}, \mathrm{NH}), 7.67$ (s, 1H, NH), 6.60-6.69 (m, 4H, Ar-H), 5.60 (s, 1H, OH), 5.10 (s, $1 \mathrm{H}, \mathrm{Ar}-\mathrm{CH}), 4.08\left(\mathrm{q}, 1 \mathrm{H}, \mathrm{OCH}_{2} \mathrm{CH}_{3}, J=7.8 \mathrm{~Hz}\right), 2.24(\mathrm{~s}, 3 \mathrm{H})$, $1.12\left(\mathrm{t}, 3 \mathrm{H}, \mathrm{OCH}_{2} \mathrm{CH}_{3}, J=7.0 \mathrm{~Hz}\right) .{ }^{13} \mathrm{C} \mathrm{NMR}$ (DMSO- $\left.d_{6}\right): \delta$ 167.2, 160.3, 158.3, 147.7, 144.6, 130.0, 119.6, 113.9, 112.6, 104.2, 61.7, 54.9, 14.05, 17.7. FTIR (KBr): $3238(\mathrm{NH}), 3113$ $(\mathrm{OH}), 2980,2835,1732(\mathrm{C}=\mathrm{O}$, ester $), 1649(\mathrm{C}=\mathrm{O}$, Pyrimidine), 1620 and 1460 (Aromatic), 1510, 1460, 1282, $1176,786 \mathrm{~cm}^{-1}$.

\subsubsection{Ethyl 4- (4'-hydroxyphenyl)-6-methyl-2-oxo-1, 2, 3, 4-tetrahydropyrimidine-5-carboxylate}

Yield: $0.42 \mathrm{~g}(60 \%)$. m. p: $226-228^{\circ} \mathrm{C}$ (Lit. m. p: $227-$ $\left.229^{\circ} \mathrm{C}\right) .{ }^{1} \mathrm{H}$ NMR (DMSO-d $\left.d_{6}\right): \delta 9.32(\mathrm{~s}, 1 \mathrm{H}, \mathrm{NH}), 7.67$ (s, 1 $\mathrm{H}, \mathrm{NH}), 6.69-7.02$ (m, $4 \mathrm{H}, \mathrm{Ar}-\mathrm{H}), 5.61$ (s, 1H, OH), 5.04 (s, $1 \mathrm{H}, \mathrm{Ar}-\mathrm{CH}$ ), 3.97 (q, $2 \mathrm{H}, \mathrm{OCH}_{2} \mathrm{CH}_{3}, J=7.2 \mathrm{~Hz}$ ), 2.23 (s, 3 $\mathrm{H}), 1.08$ (t, $\left.3 \mathrm{H}, \mathrm{OCH}_{2} \mathrm{CH}_{3}, J=7.2 \mathrm{~Hz}\right) .{ }^{13} \mathrm{C} \mathrm{NMR}$ (DMSO-d ${ }_{6}$ ): $\delta 165.4,156.6,152.2,147.8,135.5,128.9,128.4127 .4$, 115.0, 99.8, 59.1, 53.5, 17.8, 14.1. FTIR (KBr): $3417(\mathrm{NH})$, $3241(\mathrm{OH}), 3120,2984,1742(\mathrm{C}=\mathrm{O}$, ester $), 1687(\mathrm{C}=\mathrm{O}$, Pyrimidine), 1612 and 1485 (Aromatic) $\mathrm{cm}^{-1}$.

\subsubsection{Ethyl 6-methyl-2-oxo-4- (thiophene-2'-yl)-1, 2, 3, 4-tetrahydropyrimidine-5-carboxylate}

Yield: $0.38 \mathrm{~g}(57 \%)$. m. p: $207-209^{\circ} \mathrm{C}$ (Lit. m. p: 209$210^{\circ} \mathrm{C}$ ). ${ }^{1} \mathrm{H}$ NMR (DMSO- $\left.d_{6}\right): \delta 8.51(\mathrm{~s}, 1 \mathrm{H}, \mathrm{NH}), 6.87-6.95$ (m, 2H, Ar-H), 7.17 (s, 1H, NH), 6.35 (s, 1H, Ar-H), 5.68 (s, $1 \mathrm{H}, \mathrm{ArCH}), 4.13$ (q, 2H, $\mathrm{OCH}_{2} \mathrm{CH}_{3}, J=7.2 \mathrm{~Hz}$ ), 2.27 (s, 3H), $1.29\left(\mathrm{t}, 3 \mathrm{H}, \mathrm{OCH}_{2} \mathrm{CH}_{3}, J=7.0 \mathrm{~Hz}\right) .{ }^{13} \mathrm{C} \mathrm{NMR}$ (DMSO- $\left.d_{6}\right): \delta$ $165.4,153.9,147.3,146.8,126.7,124.8,123.9,101.6,60.2$, 50.6, 18.5, 14.2. FTIR (KBr): $3340(\mathrm{NH}), 3240,3120,1738$ 
$(\mathrm{C}=\mathrm{O}$, ester $), 1650(\mathrm{C}=\mathrm{O}$, Pyrimidine $), 1630 \mathrm{~cm}^{-1}$.

\subsubsection{Ethyl 6-methyl-4-phenyl-2-thioxo-1, 2, 3, 4-tetrahydropyrimidine-5-carboxylate}

Yield: $0.4 \mathrm{~g}(72 \%)$. m. p: $207-209^{\circ} \mathrm{C}$ (Lit. m. p: $209-$ $211^{\circ} \mathrm{C}$ ). ${ }^{1} \mathrm{H}$ NMR (DMSO- $\left.d_{6}\right): \delta 7.27-7.31(\mathrm{~m}, 5 \mathrm{H}, \mathrm{Ph}), 5.30$ (s, 1H, CH, Ar-CH), 4.07 (q, 2H, $\mathrm{OCH}_{2} \mathrm{CH}_{3}, J=7.32 \mathrm{~Hz}$ ), $2.44(\mathrm{~s}, 1 \mathrm{H}, \mathrm{NH}), 2.34\left(\mathrm{~s}, 3 \mathrm{H}, \mathrm{CH}_{3}\right), 2.15(\mathrm{~s}, 1 \mathrm{H}, \mathrm{NH}), 1.16$ (t, $3 \mathrm{H}, \mathrm{OCH}_{2} \mathrm{CH}_{3}, J=7.23 \mathrm{~Hz}$ ). ${ }^{13} \mathrm{C}$ NMR (DMSO- $d_{6}$ ): $\delta$ $176.0,166.9,145.6,144.5,129.5,128.8,127.6,127.4,126.2$ 102.8, 61.0, 56.1, 17.8, 14.5. FTIR (KBr): $3327(\mathrm{NH}), 3174$, 3105, 2981, 1745 (C=O, ester), 1622 and 1498 (Aromatic), $1571(\mathrm{C}=\mathrm{C}), 1465,1326,1197,759 \mathrm{~cm}^{-1}$.

\subsubsection{Ethyl 4- (2', 4'-dichlorophenyl)-6-methyl-2-thioxo-1, \\ 2, 3, 4-tetrahydropyrimidine-5-carboxylate}

Yield: $0.5 \mathrm{~g}(58 \%)$ m. p: $224-226^{\circ} \mathrm{C}$ (Lit. m. p: 226$228^{\circ} \mathrm{C}$ ). ${ }^{1} \mathrm{H}$ NMR (DMSO- $\left.d_{6}\right): \delta$ 7.25-7.36 (m, 3H, Ar-H), 5.37 (s, $1 \mathrm{H}, \mathrm{Ar}-\mathrm{CH}), 4.09$ (q, $\left.2 \mathrm{H}, \mathrm{OCH}_{2} \mathrm{CH}_{3}, J=7.2 \mathrm{~Hz}\right), 2.61$ $(\mathrm{s}, 1 \mathrm{H}, \mathrm{NH}), 2.36\left(\mathrm{~s}, 3 \mathrm{H}, \mathrm{CH}_{3}\right), 2.10(\mathrm{~s}, 1 \mathrm{H}, \mathrm{NH}), 1.19(\mathrm{t}, 3 \mathrm{H}$, $\mathrm{OCH}_{2} \mathrm{CH}_{3}, J=4.68 \mathrm{~Hz}$ ). ${ }^{13} \mathrm{C}$ NMR (DMSO- $d_{6}$ ): $\delta \quad 174.4$, $167.2,160.3,140.9,133.7,133.2,130.2,129.8,126.8,104.2$, 61.7, 45.5, 17.7, 14.0. FTIR (KBr): $3359(\mathrm{NH}), 1739(\mathrm{C}=\mathrm{O}$, ester), 1695, 1624 and 1498 (Aromatic) $\mathrm{cm}^{-1}$.

\subsubsection{Ethyl 4- (4'-hydroxyphenyl)-6-methyl-2-thioxo-1, 2, 3, 4-tetrahydropyrimidine-5-carboxylate}

Yield: $0.45 \mathrm{~g}(61 \%)$. m. p: $200-202^{\circ} \mathrm{C}$ (Lit. m. p: $202-$ $\left.203^{\circ} \mathrm{C}\right)$. FTIR (KBr): $3245(\mathrm{NH}), 3187(\mathrm{OH}), 1745(\mathrm{C}=\mathrm{O}$, ester), $1679,1642 \mathrm{~cm}^{-1} .{ }^{1} \mathrm{H}$ NMR (DMSO- $d_{6}$ ): $=\delta 6.94-7.45$ (m, $4 \mathrm{H}, \mathrm{Ar}-\mathrm{H}), 5.27$ (s, $1 \mathrm{H}, \mathrm{Ar}-\mathrm{CH}), 4.24\left(\mathrm{q}, 2 \mathrm{H}, \mathrm{OCH}_{2} \mathrm{CH}_{3}\right.$, $J=7.15 \mathrm{~Hz}), 5.13(1 \mathrm{H}, \mathrm{OH}), 2.46(\mathrm{~s}, 1 \mathrm{H}, \mathrm{NH}), 2.14(\mathrm{~s}, 1 \mathrm{H}$, $\mathrm{NH}), 1.91\left(\mathrm{~s}, 3 \mathrm{H}, \mathrm{CH}_{3}\right), 1.30\left(\mathrm{t}, 3 \mathrm{H}, \mathrm{OCH}_{2} \mathrm{CH}_{3}, J=7.15 \mathrm{~Hz}\right)$. ${ }^{13} \mathrm{C}$ NMR (DMSO- $d_{6}$ ): $\delta 167.2,156.5,150.3,147.2,135.9$, $128.4,126.4,119.7,115.7,106.4,61.7,49.2,14.9,14.4$.

\subsubsection{Ethyl 4- (3'-hydroxyphenyl)-6-methyl-2-thioxo-1, 2, 3, 4-tetrahydropyrimidine-5-carboxylate}

Yield: $0.46 \mathrm{~g}(63 \%)$. m. p: $185-187^{\circ} \mathrm{C}$ (Lit. m. p: $184-$ $\left.186^{\circ} \mathrm{C}\right) .{ }^{1} \mathrm{H}$ NMR (DMSO- $\left.d_{6}\right): \delta 7.66(\mathrm{~s}, 1 \mathrm{H}, \mathrm{Ar}-\mathrm{H}), 6.58-$ $7.15(\mathrm{~m}, 3 \mathrm{H}, \mathrm{Ar}-\mathrm{H}), 5.67(\mathrm{~s}, 1 \mathrm{H}, \mathrm{OH}), 5.04(\mathrm{~s}, 1 \mathrm{H}, \mathrm{Ar}-\mathrm{CH})$, 4.05 (q, $\left.2 \mathrm{H}, \mathrm{OCH}_{2} \mathrm{CH}_{3}, J=7.0 \mathrm{~Hz}\right), 2.74(\mathrm{~s}, 1 \mathrm{H}, \mathrm{NH}), 2.02$ (s, $1 \mathrm{H}, \mathrm{NH}), 2.22\left(\mathrm{~s}, 3 \mathrm{H}, \mathrm{CH}_{3}\right), 1.10\left(\mathrm{t}, 3 \mathrm{H}, \mathrm{OCH}_{2} \mathrm{CH}_{3}\right.$, $J=7.0 \mathrm{~Hz}$ ). ${ }^{13} \mathrm{C}$ NMR (DMSO- $d_{6}$ ): $\delta 174.4,168.3,160.5$, 159.3, 147.2, 135.9, 128.4, 119.7, 115.7, 106.4, 61.7, 49.2, 14.5, 14.4. FTIR (KBr): $3363(\mathrm{NH}), 3171(\mathrm{OH}), 3082,2993$, $1728(\mathrm{C}=\mathrm{O}$, ester $), 1560(\mathrm{C}=\mathrm{C}), 1485,1329,1153,1091$, $765 \mathrm{~cm}^{-1}$

\subsubsection{5-acetyl-6-methyl-4- (pyridine-3'-yl)-3, 4-dihydropyrimidine-2 (1H)-one}

Yield: $0.34 \mathrm{~g}(54 \%)$. m. p: $210-212^{\circ} \mathrm{C} .{ }^{1} \mathrm{H}$ NMR (DMSO$\left.d_{6}\right): \delta 8.43(\mathrm{~s}, 1 \mathrm{H}, \mathrm{NH}), 7.60-7.85(\mathrm{~m}, 4 \mathrm{H}, \mathrm{Ar}-\mathrm{H}), 7.47(\mathrm{~s}, 1 \mathrm{H}$, $\mathrm{NH}), 5.30(\mathrm{~s}, 1 \mathrm{H}, \mathrm{Ar}-\mathrm{CH}), 2.30\left(\mathrm{~s}, 3 \mathrm{H}, \mathrm{CH}_{3}\right), 2.15(\mathrm{~s}, 3 \mathrm{H}$, $\left.\mathrm{CH}_{3}\right) .{ }^{13} \mathrm{C}$ NMR (DMSO- $\left.d_{6}\right): \delta 196.5,150.6,150.3,147.2$ 145.0, 134.4, 133.7, 123.4, 106.5, 47.7, 27.1, 15. FTIR $(\mathrm{KBr}): 3187(\mathrm{NH}), 1694(\mathrm{C}=\mathrm{O}), 1610(\mathrm{C}=\mathrm{O}$, Pyrimidine $)$, $1586(\mathrm{C}=\mathrm{C}), \mathrm{cm}^{-1}$.

\subsubsection{5-acetyl-4- (2'-methoxyphenyl)-6-methyl-3, 4-dihydropyrimidine-2 (1H)-one}

Yield: $0.35 \mathrm{~g}(56 \%)$. m. p: $285-287^{\circ} \mathrm{C}$ (Lit. m. p: $287-$ $\left.288^{\circ} \mathrm{C}\right) .{ }^{1} \mathrm{H}$ NMR $\left(\right.$ DMSO- $\left.d_{6}\right):=\delta 9.12(\mathrm{~s}, 1 \mathrm{H}, \mathrm{NH}), 7.12(\mathrm{~s}$, $1 \mathrm{H}, \mathrm{NH}), 6.85-7.21(\mathrm{~m}, 4 \mathrm{H}, \mathrm{Ar}-\mathrm{H}), 3.77\left(\mathrm{~s}, 3 \mathrm{H}, \mathrm{OCH}_{3}\right), 5.37$ (s, $1 \mathrm{H}, \mathrm{Ar}-\mathrm{CH}), 2.32\left(\mathrm{~s}, 3 \mathrm{H}, \mathrm{CH}_{3}\right), 2.09\left(\mathrm{~s}, 3 \mathrm{H}, \mathrm{CH}_{3}\right) .{ }^{13} \mathrm{C}$ NMR (DMSO- $d_{6}$ ): $\delta 199.5,156.3,150.3,147.2,128.0$, 127.4, 121.3, 120.9, 114.1, 107.5, 56.2, 38.7, 27.1, 15.4. FTIR (KBr): $3264(\mathrm{NH}), 2926,1710(\mathrm{C}=\mathrm{O}), 1675(\mathrm{C}=\mathrm{O}$, Pyrimidine $), 1590(\mathrm{C}=\mathrm{C}) \mathrm{cm}^{-1}$.

\subsubsection{5-acetyl-4- (2', 4'-dichlorophenyl)-6-methyl-3, 4-dihydropyrimidine-2 $(1 \mathrm{H})$-one}

Yield: $0.38 \mathrm{~g}(52 \%)$. m. p: $240-242^{\circ} \mathrm{C}$ (Lit. m. p: $242-$ $244^{\circ} \mathrm{C}$ ). ${ }^{1} \mathrm{H}$ NMR (DMSO- $d_{6}$ ): $\delta 9.13(\mathrm{br} \mathrm{s}, 1 \mathrm{H}, \mathrm{NH}), 7.31$ (s, 1H, Ar-H), 7.12-7.24 (m, 2H, Ar-H), 6.98 (br s, $1 \mathrm{H}, \mathrm{NH}$ ), $5.63(\mathrm{~s}, 1 \mathrm{H}, \mathrm{Ar}-\mathrm{CH}), 3.55\left(\mathrm{~s}, 3 \mathrm{H}, \mathrm{CH}_{3}\right), 2.33\left(\mathrm{~s}, 3 \mathrm{H}, \mathrm{CH}_{3}\right)$. ${ }^{13} \mathrm{C}$ NMR (DMSO- $\left.d_{6}\right): \delta 197.5,150.6,145.08,140.2,134.2$, 133.7, 130.4, 129.8, 126.8, 105.8, 39.5, 27.4, 15.6. FTIR $(\mathrm{KBr}): 3380(\mathrm{NH}), 3230,3104,2960,1700(\mathrm{C}=\mathrm{O}), 1641$ $\left(\mathrm{C}=\mathrm{O}\right.$, Pyrimidine), $1613 \mathrm{~cm}^{-1}$.

\subsubsection{5-acetyl-4- (2', 6'-dichlorophenyl)-6-methyl-3, 4-dihydropyrimidine-2 (1H)-one}

Yield: $0.25 \mathrm{~g}(34 \%)$. m. p: $250-252^{\circ} \mathrm{C} .{ }^{1} \mathrm{H}$ NMR (DMSO$\left.d_{6}\right): \delta 9.1(\mathrm{~s}, 1 \mathrm{H}, \mathrm{NH}), 7.51(\mathrm{~s}, 1 \mathrm{H}, \mathrm{NH}), 7.34-7.12(\mathrm{~m}, 3 \mathrm{H}$, Ar-H), 6.1 (s, 1H, CH), 2.25 (s, 3H, $\left.\mathrm{CH}_{3}\right), 2.04\left(\mathrm{~s}, 3 \mathrm{H}, \mathrm{CH}_{3}\right)$. ${ }^{13} \mathrm{C}$ NMR (DMSO- $d_{6}$ ): $\delta 199.5,150.3,146.2,145.0,134.2$, $133.7,127.4,127.2,126.8,106.8,29.5,27.1,15.8$. FTIR $(\mathrm{KBr}): 3095(\mathrm{NH}), 2927,1717 \quad(\mathrm{C}=\mathrm{O}), 1659 \quad(\mathrm{C}=\mathrm{O}$, Pyrimidine), $1577(\mathrm{C}=\mathrm{C}) \mathrm{cm}^{-1}$.

\subsubsection{5-acetyl-6-methyl-4- (thiophene-2'-yl)-3, 4-dihydropyrimidine-2 (1H)-one}

Yield: $0.39 \mathrm{~g}(66 \%)$. m. p: $190-192^{\circ} \mathrm{C}{ }^{1} \mathrm{H}$ NMR (DMSO$\left.d_{6}\right): \delta 9.03(\mathrm{~s}, 1 \mathrm{H}, \mathrm{NH}), 7.56(\mathrm{~s}, 1 \mathrm{H}, \mathrm{NH}), 6.52-6.81(\mathrm{~m}, 3 \mathrm{H}$, $\mathrm{Ar}-\mathrm{H}), 5.26(\mathrm{~s}, 1 \mathrm{H}, \mathrm{Ar}-\mathrm{CH}), 2.49\left(\mathrm{~s}, 3 \mathrm{H}, \mathrm{CH}_{3}\right), 2.16(\mathrm{~s}, 3 \mathrm{H}$, $\mathrm{CH}_{3}$ ). ${ }^{13} \mathrm{C}$ NMR (DMSO- $d_{6}$ ): $\delta 197.5,150.3,145.01,142.2$, 127.4, 126.2, 124.8, 105.8, 48.6, 26.6, 15.2. FTIR (KBr): $3264(\mathrm{NH}), 2926,1730(\mathrm{C}=\mathrm{O}), 1675(\mathrm{C}=\mathrm{O}$, Pyrimidine $)$ $1590(\mathrm{C}=\mathrm{C}), 1448 \mathrm{~cm}^{-1}$.

\section{Results and Discussion}

There are many new methodologies for the synthesis of substituted Dihydropyrimidones. Very advance work has been done on Biginelli reaction by different research groups. But all the variabilities are majorly in the use of catalysts. In 1989 aliphatic aldehydes were used instead of aromatic aldehydes [19]. However, it has been seen that many organic solvents and the catalysts employed are not always environmentally friendly, and many catalysts used are uncommon or exotic, to say the least.

In our work with Biginelli reaction, we have earlier used different catalysts and solvents [13-17]. This encouraged us to initiate a methodical study to look into the feasibility of solvent-free and catalyst-free reactions under modified experimental conditions towards the development of a green 
methodology for useful scaffolds.

In the following efforts at developing some "green" protocol is presented. The results of various experiments are given. The reactions were carried out at a controlled temperature range. Various products 2.2.1-2.2.16 have been prepared using this solvent and catalyst-free conditions and the products isolated during the present work compared well with the reported in the literature (M. P, FTIR, and NMR spectra) (Scheme 1, Table 1).

$$
\overbrace{\mathrm{R}}^{\text {Heat } 120-130^{\circ} \mathrm{C}}
$$

Figure 1. General synthetic approach to pyrimidones and thiones.

${ }^{1}$ HNMR spectra of compound 1-11, where ethyl acetoacetate was used as active methylene compound showed triplet for the ester methyl at 1.00-1.30 ppm $\left(\mathrm{OCH}_{2} \mathrm{CH}_{3}\right)$ and a quartet of methylene protons at 3.90-4.13 ppm $\left(\mathrm{OCH}_{2} \mathrm{CH}_{3}\right)$. Methyl group showed a singlet in different cases in between 1.91 to $2.36 \mathrm{ppm}$. A singlet at 5.04-5.68 ppm was due to proton at position 4 of the pyrimidine ring. The two different broad signals around 9.2 and $7.7 \mathrm{ppm}$ were assigned to the resonance of two $\mathrm{NH}$ protons of the pyrimidine rings. Other protons of the aryl group were observed in the aromatic region.

In the ${ }^{13} \mathrm{C} \mathrm{NMR}$, methyl carbon $\left(-\mathrm{OCH}_{2} \mathrm{CH}_{3}\right)$ of the ester group showed signals near $14 \mathrm{ppm}$ and methylene carbons ($\mathrm{OCH}_{2} \mathrm{CH}_{3}$ ) around $60 \mathrm{ppm}$ due to its attachment with oxygen. The carbon of the pyrimidine rings where a methyl group is attached shows signals near $148 \mathrm{ppm}$. A peak near $152 \mathrm{ppm}$ represents pyrimidine carbonyl carbon and that of around 165 ppm, represents ester carbonyl.

FTIR spectra were also consistent with the structure reported in the literature. Carbonyl $(\mathrm{C}=\mathrm{O})$ of the ester group showed absorption at $1725-1740 \mathrm{~cm}^{-1}, \mathrm{NH}$ at $3260-3270 \mathrm{~cm}^{-}$ ${ }^{1}$, and carbonyl $(\mathrm{C}=\mathrm{O})$ of pyrimidine ring at $1680 \mathrm{~cm}^{-1}$. Peaks at $1620 \mathrm{~cm}^{-1}$ and $1490 \mathrm{~cm}^{-1}$ represented the presence of an aromatic ring in the compound.

In reactions 12-16 where acetylacetone is used instead of ethyl acetoacetate give proton signals in between 2.09 to 2.50 ppm for methyl of the acetyl group.

Table 1. Catalyst free, solvent free Biginelli products.

\begin{tabular}{|c|c|c|c|c|c|c|}
\hline \multirow{2}{*}{ Compound No. } & \multirow{2}{*}{ Ar } & \multirow{2}{*}{$\mathbf{R}$} & \multirow{2}{*}{$\mathbf{X}$} & \multirow{2}{*}{ Yield (\%) } & \multicolumn{2}{|c|}{ Melting point $\left({ }^{\circ} \mathrm{C}\right)$} \\
\hline & & & & & Found & Reported \\
\hline 1 & $\mathrm{C}_{6} \mathrm{H}_{5}$ & OEt & $\mathrm{O}$ & 72 & $200-202$ & $202-204^{21}$ \\
\hline 2 & $2-\mathrm{CH}_{3} \mathrm{OC}_{6} \mathrm{H}_{4}$ & OEt & $\mathrm{O}$ & 67 & $257-259$ & $259-260^{22}$ \\
\hline 3 & $2,4-\mathrm{ClC}_{6} \mathrm{H}_{4}$ & OEt & $\mathrm{O}$ & 59 & $249-251$ & $249-250^{23}$ \\
\hline 4 & Pyridine-3-yl & $\mathrm{OEt}$ & $\mathrm{O}$ & 66 & $215-217$ & $217-218^{24}$ \\
\hline 5 & $3-\mathrm{HOC}_{6} \mathrm{H}_{4}$ & OEt & $\mathrm{O}$ & 57 & $162-164$ & $164-166^{22}$ \\
\hline 6 & $4-\mathrm{HOC}_{6} \mathrm{H}_{4}$ & OEt & $\mathrm{O}$ & 60 & $226-228$ & $227-229^{21}$ \\
\hline 8 & $\mathrm{C}_{6} \mathrm{H}_{5}$ & OEt & $\mathrm{S}$ & 72 & $207-209$ & $209-211^{20}$ \\
\hline 9 & $2,4-\mathrm{ClC}_{6} \mathrm{H}_{4}$ & OEt & $\mathrm{S}$ & 58 & $224-226$ & $225-227^{25}$ \\
\hline 10 & $4-\mathrm{HOC}_{6} \mathrm{H}_{4}$ & $\mathrm{OEt}$ & $\mathrm{S}$ & 61 & $200-202$ & $202-203^{26}$ \\
\hline 11 & $3-\mathrm{HOC}_{6} \mathrm{H}_{4}$ & OEt & $\mathrm{S}$ & 63 & $185-187$ & $184-186^{22}$ \\
\hline 12 & Pyridine-3-yl & $\mathrm{Me}$ & $\mathrm{O}$ & 54 & $210-212$ & ----- \\
\hline 13 & $2-\mathrm{CH}_{3} \mathrm{OC}_{6} \mathrm{H}_{4}$ & $\mathrm{Me}$ & $\mathrm{O}$ & 56 & $285-287$ & $287-288^{20}$ \\
\hline 14 & $2,4-\mathrm{ClC}_{6} \mathrm{H}_{4}$ & $\mathrm{Me}$ & $\mathrm{O}$ & 52 & $240-242$ & $242-244^{22}$ \\
\hline 15 & $2,6-\mathrm{ClC}_{6} \mathrm{H}_{4}$ & $\mathrm{Me}$ & $\mathrm{O}$ & 34 & $250-252$ & ---- \\
\hline
\end{tabular}

The yields of the above described reactions are in most cases in between 60 to $70 \%$ which is comparable to or higher than the yields obtained by using different catalysts and solvents as listed in Table 1. The yields were not optimized and are based on the recrystallized products. However, it may be enhanced by increasing temperature or reaction time.

\section{Conclusion}

In conclusion, we have optimized and developed as ecofriendly, solvent free, catalyst free conditions Biginilli reaction to synthesize dihydropyrimidones (-thiones) (DHPMs) in variable yields. A series of DHPMs has been synthesized in good to excellent yield via easy separation to show the significance of methodology. Further, the Biginilli reaction will be diversely to access more compounds to explore their biological potential to find lead as new therapeutic agents.

\section{Acknowledgements}

Work from the author's laboratory was supported by grants from the Higher Education Commission of Pakistan.

\section{References}

[1] Rovnyak, G. C.; Kimball, S. D.; Beyer. B.; Cucinotta, G.; DiMarco, J. D.; Gougoutas, J.; Hedberg, A.; Malley, M.; McCarthy, J. P.; Zhang, R.; J. Med Chem. 1995, 38, 119.

[2] Kappe, C. O.; Fabian, W. M. F.; Semones, M. A. Tetrahedron Lett. 1997, 53, 2803.

[3] Kappe, C. O. Eur. J. Med. Chem. 2000, 35, 1043. 
[4] Overman, L. E.; Rabinowitz, M. H.; Renhowe, P. A.; J. Am. Chem Soc. 1995, 117, 2657.

[5] Ashok, D.; Patil, N.; Vasant K.; Wilhelmus, C. K.; Mark, F. B.; Alan, J. F.; Charles, D. B.; Shing, M.; Alemseged, T.; Brad, C.; J. Org. Chem. 1995, 60, 1182.

[6] Snider, B.; Chen, J.; Patil, A. D.; Freyer, A.; Tet. Lett. 1996, $37,6977$.

[7] Rao, A. V. R.; Gurjar, M. K.; Vasudevan, J.; J. Chem. Soc, Chem. Comm., 1995, 1369.

[8] Folkers, K.; Johnson, T. B.; J. Am. Chem. Soc. 1933, 7, 55, 2886.

[9] Folkers, K.; Johnson, T. B.; J. Am. Chem. Soc. 1933, 9, 55, 3784 .

[10] Kappe, C. O.; J. Org. Chem. 1997, 62, 7201.

[11] Hu, E. H.; Sidler, D. R.; Dolling, U. H.; J. Org. Chem. 1998, $63,3454$.

[12] Ghafoor, A.; Sajid, N.; Mukhtar, A.; Ali, S.; Aslam, S.; Shahi, M. N.; Adnan, M.; Khan M. A.; Asian J. Chem. 2016, 28, 23.

[13] Karamat, A.; Khan, M. A.; Sharif, A.; J. Chin. Chem. Soc. 2010, 57, 1099.

[14] Imtiaz, S.; Khan, M. A.; Sharif, A.; Ahmad, E.; Lin, W. O.; Munawar, M. A.; J. Chin. Chem. Soc. 2012, 59, 1146.

[15] Zafar, A. M.; Qureshi, S.; Khan, M. N.; Azad, M.; Munawar, M. A.; Khan, M. A.; Asian J. Chem.; 2013, 25, 3244.
[16] Noreen, S.; Perveen, S.; Khan, M. N.; Nazeer, A.; Khan, M. A.; Munawar, M. A. Babar, R.; Sohail, F.; Azad, M.; Bernardino, A. M. R.; Dos Santos, M. S.; Asian J. Chem. 2013, 25, 4770.

[17] Nazeer, A.; Ain, Q.; Kanwal, F.; Khan, M. N.; Perveen, S.; Amina, H.; Lin, W. O.; Uzzaman, A.; Adnan, M.; Khan, M. A.; Affinidad. 2015. 72, 60.

[18] Hatamjafari, F.; Nezhad, F. G.; Orient. J. Chem. 2014. 30, 355.

[19] Fringuelli, F.; Girotti, R.; Piermatti, O.; Pizzo, F.; Vaccaro, L.; Org. Lett. 2006, 8, 5741.

[20] Aridoss, G.; Jeong, Y. T.; Bull. Korean Chem. Soc. 2010. 31, 863.

[21] Lu, J.; Bai, Y.; Synthesis. 2002, 4, 466.

[22] Salehi, P.; Dabiri, M.; Zolfigol, M. A.; Fard, M. A.; Tet. Lett. 2003, 44, 2889.

[23] zizian, J.; Mohammadi, A. A.; Karimi, A. R.; Mohammadizadeh, M. R..; Appl. Catal. A. 2006, 300, 85.

[24] Russowsky, D.; Lopes, F. A.; Da Silva, V. S. S.; Canto, K. F. S.; D'Oca, M. G. M.; Godoi. M. N.; J. Braz. Chem. Soc.; 2004 15,165 .

[25] Wang, L.; Qian, C.; Tian, H.; Ma, Y.; Syn. Comm. 2003, 33, 1468 .

[26] Su, W.; Li, J.; Zheng, Z.; Shen, Y.; Tet. Lett. 2005, 46, 6037.

[27] Bose, D. S.; Fatima, L.; Mereyala, H. B.; J. Org. Chem. 2003, 68, 587. 